\title{
Clinical and economic outcomes of adjunctive therapy with pregabalin or usual care in generalized anxiety disorder patients with partial response to selective serotonin reuptake inhibitors
}

Enrique Álvarez ${ }^{*}$, José M Olivares², José L Carrasco ${ }^{3}$, Vanessa López-Gómez ${ }^{4}$ and Javier Rejas ${ }^{5}$

\begin{abstract}
Background: This study is done to compare the effect of adjunctive therapy with pregabalin versus usual care (UC) on health-care costs and clinical and patients consequences in generalized anxiety disorder (GAD) subjects with partial response (PR) to a previous selective serotonin reuptake inhibitor (SSRI) course in medical practice in Spain.

Methods: Post hoc analysis of patients with PR to SSRI monotherapy enrolled in a prospective 6-month naturalistic study was done. PR was defined as a Clinical Global Impression (CGI) scale score $\geq 3$ and insufficient response with persistence of anxiety symptoms $\geq 16$ in the Hamilton Anxiety Rating Scale (HAM-A). Two groups were analyzed: 1) adjunctive therapy (AT) with pregabalin (150-600 mg/day) to existing therapy and 2) UC (switching to a different SSRI or adding another anxiolytic different than pregabalin). Costs included GAD-related health-care resources utilization. Consequences were a combination of psychiatrist-based measurements [HAM-A, CGl, and Montgomery-Asberg Depression Rating Scale (MADRS)] and patient-reported outcomes [Medical Outcomes Study Sleep (MOS-sleep) scale, disability (World Health Organization Disability Assessment Schedule II (WHO-DAS II) and quality-of-life (Euro Qol-5D (EQ-5D)]. Changes in both health-care costs and scale scores were compared separately at end-of-trial visit by a general linear model with covariates.
\end{abstract}

Results: Four hundred eighty-six newly prescribed pregabalin and 239 UC GAD patients [mean (SD) HAM-A 26.7 (6.9) and CGI $4.1(0.5)$ ] were analyzed. Adding pregabalin was associated with significantly higher mean $(95 \% \mathrm{Cl})$ score reductions vs. UC in HAM-A $[-14.9(-15.6 ;-14.2)$ vs. $-11.2(-12.2 ;-10.2), p<0.001]$ and MADRS $[-11.6(-12.2 ;-10.9)$ vs. $-7.8(-8.7 ;-6.8), p<0.001]$. Changes in all patient-reported outcomes favored significantly patients receiving pregabalin, including quality-of-life gain; $26.4(24.7 ; 28.1)$ vs. $19.4(17.1 ; 21.6)$ in the EQ-VAS, $p<0.001$. Health-care costs were significantly reduced in both cohorts yielding similar 6 -month costs; $€ 1,565(1,426 ; 1,703)$ pregabalin and $€ 1,406(1,200 ; 1,611) \cup C, p=0.777$. The effect of sex on costs and consequences were negligible.

Conclusion: In medical practice, GAD patients with PR to SSRI experienced greater consequence improvements with adjunctive therapy with pregabalin versus UC, without increasing health-care cost. The effect of pregabalin was independent of patient gender.

Keywords: Cost analysis, Generalized anxiety disorder, Pregabalin, SSRI, Partial response, Usual care, Routine medical practice

\footnotetext{
* Correspondence: ealvarezm@santpau.cat

'Department of Psychiatry, Hospital de la Santa Creu i San Pau, Universitat Autónoma de Barcelona, CiberSam, Carrer Sant Quintí, 89, 08026 Barcelona, Spain

Full list of author information is available at the end of the article
} 


\section{Background}

Generalized anxiety disorder (GAD) is one of the most frequent mental disorders [1-3]. GAD is characterized by a chronic evolution with a relatively late-onset age (31 years as a median age) [4]. The prevalence of GAD in western societies ranges from $2.8 \%$ in Europe to $5.7 \%$ in the United States. Specifically and according to a large epidemiological report, the prevalence of GAD in Spain has been estimated to be $2 \%$ [5]. The recent existing limitations on economic resources highlight the importance of their optimal utilization and the efficient costconsequence analysis of new treatments by health policy decision makers. The most appropriate and useful method for the estimation of economic consequences in the management of anxiety disorders is the economic analysis that take into account both the overall health-care resource costs and the acquisition cost of the prescribed drugs [6].

For anxiety disorders, their considerable chronicity of symptoms, associated comorbidities and involved physical limitations, constitute a scenario that clearly determines an important impact on work productivity as well as high medical resource use by patients. As a consequence, the economic burden of GAD in current health systems has been evaluated as highly significant $[7,8]$. Specifically in Spain, the gross annual direct cost of GAD has been estimated as $€ 2$ million in a total population of 3,014 patients. Half of these total direct costs were associated with prescription drugs, but the total figure includes also physical services, laboratory analysis, and fixed costs [9]. Otherwise, overall economic costs of GAD in Spain are still scarce and have not been evaluated until very recently [10]. Frequently, the most common psychoactive drugs used in the management of GAD were anxiolytics. Within the most prescribed anxiolytics, benzodiazepines have been proved both efficacious and rapid for the treatment of GAD patients [11]. Nonetheless, recent guidelines recommend the use of benzodiazepines in a short-term period in response to some limitations that have been observed such as their associated comorbidity of depressive symptoms and common adverse events like sedation; memory and psychomotor dysfunction; increase in abuse, tolerance, or dependence; and afflictive withdrawal symptomatology [12-16]. Accordingly, the Spanish Health Ministry guidelines recommended the use of benzodiazepines for a restricted period not longer than 2-4 weeks [17].

When considering a long-term approach therapy, GAD effective treatment should be focused on selective serotonin reuptake inhibitors (SSRIs), selective serotonin-norepinephrine reuptake inhibitors (SNRIs), and pregabalin as a first choice according to current European guidelines [18]. Besides the delayed onset of SSRI/SNRI therapeutic effect [18], other disadvantages include the fact that their use in monotherapy is contraindicated in GAD patients diagnosed with comorbid bipolar disorder [19-21]. On the other hand and despite potential misuse and abuse of pregabalin pointed out by some researchers [22,23], pregabalin has been proven effective and well-tolerated as a long-term therapy option in adult patients with GAD [24,25], and, as mentioned previously, it is recommended as a first choice in the therapy of GAD by European guidelines [18]. Pregabalin acts as a calcium channel modulator that allows a relatively rapid onset of action after only 1 week, as well as a similar efficacy with that of benzodiazepines and a lower rate of discontinuation than that of benzodiazepines and SNRIs [26,27].

Current health-care resource utilization and related costs may differ depending on both the exact therapies and health-care settings. It is important to consider clinical practice to attain a real-world framework which could differ extensively from clinical trial observations [28]. Focusing on daily clinical practice needs, the aim of this study was to compare the effect of adjunctive therapy (AT) with pregabalin versus usual care (UC) on health-care costs and clinical and patients consequences in GAD subjects with partial response (PR) to a previous SSRI course in medical practice in Spain.

\section{Methods}

\section{Study design}

This is a post hoc economic analysis based on data from a previous 6-month, multicenter, prospective observational study: the Amplification of Definition of Anxiety (ADAN) study carried out between October 2007 and January 2009 in outpatient mental health centers in Spain [29]. The ADAN study was designed to elucidate the effect of broadening DSM-IV criteria for GAD and was approved by the local ethics committee of the Hospital Clínico de San Carlos (Madrid). It was conducted according to the Helsinki Declaration for research in the human being. Due to the observational design of the study, only two visits (baseline and 6 months visit) were planned. The ADAN study also assessed the use of health-care resources and related costs, which were used for the present cost analysis to compare the impact of initiating treatment with pregabalin versus usual care.

\section{Study population}

In the ADAN study, trained psychiatrists, with at least 5 years experience in mental health diseases diagnosis, were asked to select consecutive, newly diagnosed GAD patients, according to DSM-IV criteria (APA 2000) and so-called broad criteria, until the predetermined sample size was obtained [29]. Patients of both sex, aged 18 or above, who had provided their written informed consent to participate in the study, and with partial response to SSRI monotherapy were considered eligible for inclusion. 
Patients could also have been treated simultaneously with a benzodiazepine at standard doses. Partial response was defined as an insufficient response with persistence of anxiety symptoms $>16$ in the Hamilton Anxiety Rating Scale (HAM-A) [30,31] and a Clinical Global Impression scale score $>3$ determined at baseline visit [32]. Exclusion criteria included previous GAD diagnosis, inability or difficulty to understand patient-reported outcomes questionnaires written in Spanish, a score $\leq 9$ point in the HAM-A scale and a score $>35$ in the Montgomery-Asberg Depression Rating Scale. In this analysis, only patients with a diagnosis of GAD according to DSM-IV criteria were considered eligible. Two groups (based on psychiatrist judgment) were analyzed: 1) adding pregabalin (150-600 mg/ day) to existing therapy and 2) usual care (switching to a different SSRI and/or adding another anxiolytic different than pregabalin).

\section{Use of health-care resources and cost estimation}

Health-care resource utilization associated with GAD during the previous 6-month period was retrospectively collected at baseline and at the 6-month study visit, by means of a case report form which was designed ad hoc for this economic analysis. Health-care resource utilization included the following: drug utilization, medical visits and hospitalizations (from patients' medical records), and nonpharmacological treatments (recorded during patient interviews). No records of diagnostic tests were registered since this variable was considered negligible in GAD. Four categories of health-care resources utilization were established: drug treatments, non-pharmacological therapies, medical visits (psychiatrists, psychologists, general practitioner or family physicians, and emergency room visits), and days of hospitalization in psychiatry or internal medicine wards. Non-pharmacological therapies included all those treatments used in clinical practice as complementary/adjuvant (psychosocial therapy, cognitive-conductive therapy, supportive groups, and relaxation sessions) to drug treatments for GAD. Visits to primary care, emergency department, psychologist, and psychiatrist were recorded under the category "medical visits".

Costs estimation used year 2012 prices for GADrelated health-care resources utilization under the perspective of the Spanish National Health System. The costs of drugs were estimated using retail price + taxes of the cheapest generic medication or reference price from the Spanish Pharmaceutical Drug Catalogue of 2012. The cost of non-pharmacological treatments, medical visits, and hospitalizations was obtained from the eSALUD health-care costs database for 2012 [33] updated with the 2012 health-care inflation rate [34]. Finally, some non-pharmacological resources were priced according to expert opinion and/or directly from the vendor/provider. The direct mean cost at baseline and at the 6-month visit and change from baseline was calculated by multiplying the number of resources used in each period by their respective prices.

\section{Clinical and patients consequences}

Consequences in this study were a health profile based in the combination of psychiatrist-based measurements [Hamilton Anxiety Rating Scale, Clinical Global Impression-Improvement (CGI-I) scale, and MontgomeryAsberg Depression Rating Scale (MADRS)], and patientreported-outcomes [Medical Outcomes Study Sleep (MOS-sleep) scale, disability (The World Health Organization Disability Assessment Schedule II (WHODAS II)) and quality-of-life (Euro Qol-5D (EQ-5D))] [31,35-39]. The Hamilton Anxiety Rating Scale is a 14item scale, each with a score between 0 (absence) and 4 (severe) that explores the patients' degree of anxiety and that includes two subscales, one for psychic symptoms and the other for somatic symptoms [40]. The CGI-I scale is a seven-point scale that evaluates the clinician's assessment on how much the patient's illness has improved or worsened relative to a baseline state [35]. The MADRS is a ten-item diagnostic questionnaire which psychiatrists use to measure the severity of depressive episodes in patients with mood disorders [36]. The MOS-sleep scale measures the global subjective sleep disturbance perceived by the patient based on six dimensions of sleep [37]. It consists of 12 items which form six subscales or domains: sleep disturbance, snoring, awakening with shortness of breath or headache, adequacy of sleep, daily somnolence, and sleep quantity. In addition, the MOS-sleep scale provides a summary index of sleep problems through the scores of 9 of its items: the higher the score, the worse the sleep. WHO-DAS II is a disability instrument designed based on the ICF framework, assessing six domains of functioning in daily life [38]. The WHODAS II is a standardized measurement of disability for use in diverse cultural settings, translated into 16 languages to date. The WHO-DAS II domains include understanding and communicating, getting around, self-care, getting along with others, life activities, and participation in society. The EQ-5D is a standardized health-related quality of life and is a generic self-reported measure of health used frequently in clinical and economic evaluations [39]. In this five-item generic measurement of health state, the degree of impairment is assessed in five domains: mobility, self-care, daily activities, pain/discomfort, and anxiety/depression. The scores of the five items may be used to calculate a utility index, or social tariff, in the range of -0.6 through 1.0, where the higher scores represent a better health state. This instrument also includes a $20-\mathrm{cm}$ visual analog scale (EQ-VAS) ranging from $0=$ worst imaginable health state through 100 = best imaginable health state. 


\section{Statistical analysis}

Only patients that fulfilled all inclusion criteria and none of the exclusion criteria were included in the statistical analysis. Descriptive statistics were completed for the continuous variables in the study, including the assessment of central tendency and dispersion statistics with its 95\% confidence interval when possible. The KolmogorovSmirnov test was applied to check adjustment of data to a Gaussian distribution. For categorical variables, absolute and relative frequencies were calculated. A descriptive statistical analysis with values for mean and standard deviation (SD) was performed. Mann-Whitney $U$-test was used to compare continuous variables between the two groups of patients at baseline, while the $\chi^{2}$-test or the Fisher's exact test were applied for categorical data. Differences in the use of health-care resources and costs between the two treatment groups were tested using an analysis of covariance (ANCOVA), according to the published recommendations [41] with sex, age, and baseline values as covariates. The change from baseline for quantitative variables was calculated as the final value minus baseline value and is presented as the mean value and its 95\% confidence interval (CI). Adjusted changes both in health-care costs and scale scores were compared at end-of-trial visit by a general linear model with covariates. A $p$ value of less than 0.05 was considered significant. Data analysis was performed using the Statistical Analysis System (SAS 9.1).

\section{Results}

\section{Patient disposition and baseline characteristics}

A total of 725 subjects were included in this sub-analysis sample: 486 newly prescribed pregabalin and $239 \mathrm{UC}$ GAD patients [mean (SD) HAM-A 26.7 (6.9) and CGI $4.1(0.5)]$. The two study groups were well balanced with respect to demographic characteristics at baseline (Table 1). Mean age per group was 47.0 (12.6) years in the pregabalin group and 45.2 (13.6) years in the UC group. Percentage of women $(72.9 \%$ and $69.1 \%$, respectively) and work active subjects $(51.8 \%$ and $49.8 \%$, respectively) were similar in both groups. Subjects in the pregabalin group showed higher mean baseline scores of HAM-A $(27.2 \pm 6.6$ points vs. $25.7 \pm 7.2$ points in the UC group; $p=0.015)$, as well as of MADRS $(23.6 \pm 6.9$ points vs. $21.7 \pm 7.5$ points, respectively, $p<0.001)$. Psychiatrists' clinical impression (CGI) was also higher in the pregabalin group: $4.23 \pm 0.76$ vs. $3.97 \pm 0.79$ $(p=0.000)$.

\section{Anxiety and depression outcomes}

Adding pregabalin was associated with significantly higher benefit in anxiety and depression outcomes, as reflected by mean $(95 \% \mathrm{CI})$ reduction vs. UC in HAM-A [-15.2 (-16.0; -14.4) vs. $-10.7(-11.8 ;-9.5), p<0.001]$ and MADRS $[-11.8(-12.5 ;-11.1)$ vs. $-7.3(-8.3 ;-6.3)$, $p<0.001$ ] (Figure 1). No significant differences between sexes were observed in the reduction of these variables. Similarly, the change in CGI-I scale presented significant differences between groups: -1.7 in pregabalin vs. -1.2 in UC $(p<0.005)$. Figure 2 shows in detail the specific changes in the 14 anxiety-related items included in the HAM-A. Reductions in all items favored the pregabalin group with statistically significant differences for anxious mood $(p<0.05)$, tension $(p<0.05)$, fears $(p<0.05)$, intellectual $(p<0.001)$, somatic (sensory) $(p<0.01)$, gastrointestinal $(p<0.05)$, and autonomic symptoms $(p<0.05)$.

\section{Patient-reported outcomes}

As shown in Table 2, adjusted significant changes between visits were observed in all patient-reported outcomes. The detected improvements in sleep problems measured by the MOS-sleep scale (Table 2) and WHODAS II disability scale (Figure 3 ) favored differentially to patients in the group receiving pregabalin. Mean reduction $( \pm 95 \% \mathrm{CI})$ in general index sleep problems of the MOS-sleep scale was $26.4(24.7,28.1)$ in the pregabalin group and $19.6(17.3,22.0)$ in the UC group $(p<0.001)$. All items of the MOS-sleep scale reflected reductions that significantly favored the pregabalin group, except the snoring item and the optimal sleep score that accounted for non-significant lower reductions in patients treated with pregabalin (Table 2).

Likewise, Figure 3 depicts the mean adjusted reduction in WHO-DAS II disability scale domains. All disability domains presented significant differences between groups in the score reduction at the final visit. The global WHO-DAS II changes for employed patients (seven domains) was $-21.7(-23.3,-20.1)$ in the pregabalin group and $-15.3(-17.5,-13.1)$ in the UC group $(p<$ $0.0001)$, while mean reductions for unemployed patients (six domains) were calculated as $-20.6(-22.2,-19.1)$ in the pregabalin group and $-14.7(-16.7,-12.5)$ in the UC group $(p<0.0001)$.

\section{Quality of life}

At the end of the study, changes in the majority of quality-of-life outcomes also favored significantly to patients receiving pregabalin, except for the mobility and personal care items where the differences were not significant (Table 2). Global health status estimated by the social tariff reflected a mean improvement of $0.36(0.34$, $0.38)$ for pregabalin patients and $0.29(0.26,0.33)$ for UC patients $(p<0.001)$ after 6 months of the study initiation. The patient's self-assessed quality of life calculated after visual analog scale completion also shown a significant better improvement for pregabalin patients: 26.4 (24.7, $28.1)$ vs. $19.4(17.1,21.6)(p<0.001)$. 
Table 1 Demographic and baseline clinical characteristics of patients

\begin{tabular}{llll}
\hline Characteristic & $\begin{array}{l}\text { Pregabalin } \\
\end{array}$ & $\begin{array}{l}\text { Usual care } \\
\text { N=486 }\end{array}$ & $\boldsymbol{N}=\mathbf{2 3 9}$ \\
\hline Age (years), mean (SD) & $47.0(12.6)$ & $45.2(13.6)$ & 0.070 \\
Sex (female), $n(\%)$ & $325(72.9 \%)$ & $159(69.1 \%)$ & 0.307 \\
Body mass index (kg/m²), mean (SD) & $25.6(4.0)$ & $26.1(4.7)$ & 0.185 \\
Marital status, $n(\%)$ & & & 0.625
\end{tabular}

Marital status, $n(\%)$

Married or with couple

Single

Widow/er

Divorced/separated

Educational level, $n$ (\%)

No education

Primary education

Secondary education

Intermediate educational level

Higher education (university)

Others

Work status, $n(\%)$

Active

Housewife

Sick leave

Unemployed

Retired

Does not work (students)

Others

Psychiatric comorbidities, $n$ (\%)

Major depression

Panic disorder

Social anxiety

Phobias

Obsessive-compulsive disorder

Others

Medical comorbidities, $n$ (\%)

Chronic pain

Gastrointestinal disease

Cardiovascular disease

Metabolic disease

Genitourinary disease

Others

SSRI distribution by drug, $n$ (\%)

Paroxetine
Escitalopram
Sertraline
Mirtazapine

$\begin{array}{ll}307(63.2 \%) & 154(65.0 \%) \\ 105(21.6 \%) & 57(24.1 \%) \\ 25(5.1 \%) & 9(3.8 \%) \\ 49(10.1 \%) & 17(7.2 \%)\end{array}$

15 (3.1\%) 15 (6.3\%)

$183(37.7 \%) \quad 85(35.6 \%)$

$106(21.9 \%) \quad 48(20.1 \%)$

$102(21.0 \%) \quad 46(19.3 \%)$

$75(15.5 \%) \quad 43(18.0 \%)$

$4(0.8 \%) \quad 2(0.8 \%)$

251 (51.8\%) 119 (49.8\%)

$127(26.2 \%) \quad 58(24.3 \%)$

$47(9.7 \%) \quad 11(4.6 \%)$

$32(6.6 \%) \quad 18(7.5 \%)$

$19(3.9 \%) \quad 20(8.4 \%)$

$4(0.8 \%) \quad 7(2.9 \%)$

$5(1.0 \%) \quad 6(2.5 \%)$

$\begin{array}{lll}89(18.3 \%) & 41(17.2 \%) & 0.780 \\ 67(13.8 \%) & 22(9.2 \%) & 0.100 \\ 48(9.9 \%) & 16(6.7 \%) & 0.200 \\ 38(7.8 \%) & 15(6.3 \%) & 0.550 \\ 18(3.7 \%) & 9(3.8 \%) & 0.867 \\ 94(19.3 \%) & 41(17.2 \%) & 0.542\end{array}$

$230(47.3 \%) \quad 78(32.6 \%) \quad<0.001$

$97(20.0 \%) \quad 53(22.2 \%) \quad 0.552$

$48(9.9 \%) \quad 24(10.0 \%) \quad 0.889$

$25(5.1 \%) \quad 21(8.8 \%) \quad 0.084$

$26(5.6 \%) \quad 8(3.4 \%) \quad 0.312$

$86(17.7 \%) \quad 43(18.0 \%) \quad 0.996$

$\begin{array}{lll}177(36.4) & 57(23.8) & 0.001 \\ 98(20.2) & 49(20.5) & 0.994 \\ 60(12.3) & 42(17.6) & 0.160 \\ 67(13.8) & 31(13.0) & 0.852\end{array}$

Table 1 Demographic and baseline clinical characteristics of patients (Continued)

\begin{tabular}{llll}
\hline Citalopram & $52(10.7)$ & $21(8.8)$ & 0.596 \\
$\quad$ Fluoxetine & $39(8.0)$ & $39(16.3)$ & 0.007 \\
Benzodiazepine use & & & \\
$\quad$ Users, $n$ (\%) & $405(83.3)$ & $211(88.3)$ & 0.177 \\
$\quad$ Mean (SD) \# drugs & $0.98(0.58)$ & $1.06(0.56)$ & 0.151 \\
Clinical variables, mean (SD) & & & \\
HAM-A scale score & $27.2(6.6)$ & $25.7(7.2)$ & 0.015 \\
MADRS scale score & $23.6(6.9)$ & $21.7(7.5)$ & 0.001 \\
CGI-I & $4.2(0.8)$ & $4.0(0.8)$ & 0.001 \\
\hline $\begin{array}{l}\text { SD standard deviation; Cl confidence interval; HAM-A Hamilton Anxiety Rating } \\
\text { Scale; MADRS Montgomery-Asberg Depression Rating Scale; CGI-I Clinical } \\
\text { Global Impression-Improvement scale. }\end{array}$ & &
\end{tabular}

Health-care resource utilization Pharmacological treatment

The use of pharmacological resources is detailed in Table 3. The utilization pattern of anxiolytic drugs during the study was determined by the following proportions at pregabalin vs. UC group: alprazolam (28.0\% vs. $20.9 \%)$, paroxetine $(24.9 \%$ vs. $25.9 \%)$, escitalopram ( $20.0 \%$ vs. $18.4 \%)$, lorazepam ( $19.1 \%$ vs. $21.8 \%)$, and diazepam $(11.9 \%$ vs. $10.9 \%)$ as the most commonly used benzodiazepines/SSRI for the treatment of GAD patients with partial response to SSRI in routine medical practice (Table 3). Drugs used by less than 3\% of patients are not shown. Pregabalin doses used in the study were within the recommended therapeutic range ( 75 to $600 \mathrm{mg} /$ day) for this drug: mean (standard deviation) 186.2 (106.5) $\mathrm{mg}$, for a mean duration of treatment of 5.3 (1.5) months (range: 0.1 to 6.1 months). During the study, therapeutic consequences elected by physicians in GAD patients with partial response to SSRI shown a trend towards a $1 / 3$ reduction both in benzodiazepines and SSRI drugs independently of the considered treatment for replacement (pregabalin or other drugs).

\section{Non-pharmacological health-care utilization}

Table 4 shows the average non-pharmacological health resources utilization at baseline and final visits of the study as well as its change from baseline. Patients under usual care have shown non-significant changes in nonpharmacological treatment use relative to study initiation, including psychosocial therapies, cognitive-behavioral therapies, supportive groups, and relaxation sessions. In the pregabalin group, only slightly higher non-pharmacological utilization was detected for psychosocial therapy and supportive group sessions $(p<0.05$ in both). Mean medical visits, overall and by type, as well as the number of hospitalizations are shown in Table 4. After 6 months of study, the reduction in the number of all medical visits reflected significant reductions both in adjunctive therapy with 


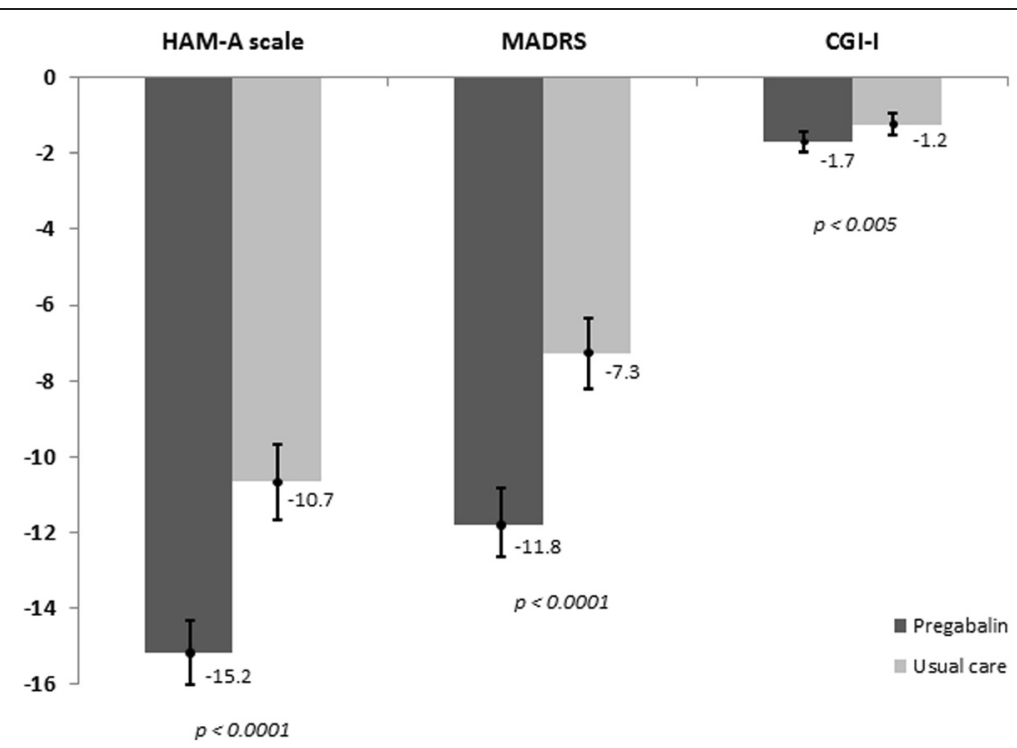

Figure 1 Mean (95\% confidence interval) reduction in clinical variables (HAM-A, MADRS, CGI-I) after 6 months of study in pregabalin and usual care groups.

pregabalin and in usual care patients. After correcting for baseline differences, the pregabalin-treated group showed non-significantly greater reductions in primary care, emergency, and psychologist visits. On the other hand, the usual care group showed slightly higher reduction in psychiatrist number of visits $(-1.3$ vs. $-1.9 ; p=0.113)$. The number of hospitalizations was similarly reduced in both groups after 6 months: -0.10 in the pregabalin-group vs. -0.09 in the UC group $(p=0.501)$.

\section{Direct costs}

Direct costs were obtained by multiplying the unit costs by the total health-care resources used in each treatment group (Table 4). The mean direct total cost at the initiation of therapy was significantly different between the two groups of patients: $€ 2,177 \pm 1,839$ for pregabalin vs. $€ 1,724 \pm 2,059$ for UC group $(p<0.001)$. This was, mainly, because of a higher baseline mean cost of medical visits in the pregabalin group $(€ 1,210 \pm 1,253$ vs. $€ 936 \pm 1,136, p<0.001)$ which was the main component

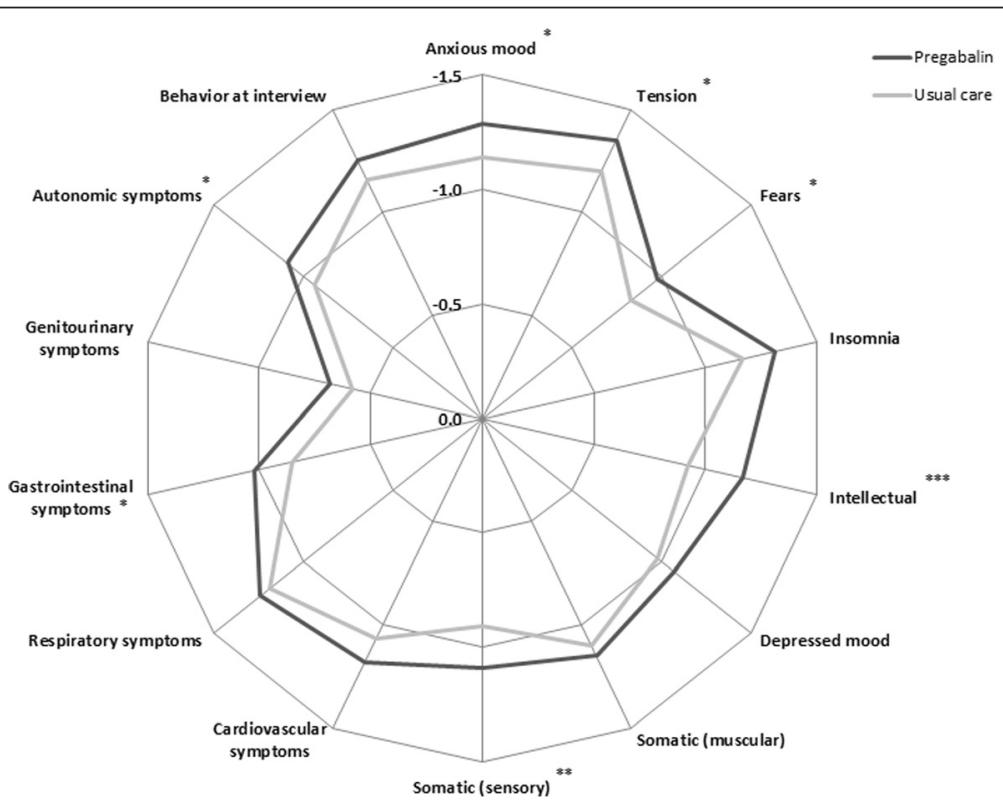

Figure 2 Adjusted mean reduction in the raw score of 14 individual items of HAM-A after 6 months of study, by treatment group. ${ }^{*} p<0.05$; ${ }^{* *} p<0.01$; ${ }^{* *} p<0.001$. 
Table 2 MOS-sleep and EQ-5D questionnaire scores by treatment group

\begin{tabular}{|c|c|c|c|c|c|c|c|c|}
\hline & \multicolumn{3}{|l|}{ Pregabalin } & \multicolumn{3}{|l|}{ Usual care } & \multirow{2}{*}{\multicolumn{2}{|c|}{$\begin{array}{l}p \text { between } \\
\text { groups }\end{array}$}} \\
\hline & \multicolumn{3}{|l|}{$N=486$} & \multicolumn{3}{|l|}{$N=239$} & & \\
\hline & Baseline & Final & Change (95\% Cl) & Baseline & Final & Change $(95 \% \mathrm{Cl})$ & Baseline & Final $^{\mathrm{a}}$ \\
\hline \multicolumn{9}{|l|}{ MOS-sleep scale, mean (SD) } \\
\hline Sleep disturbance (0-100) & $58.1(18.9)$ & $27.1(17.5)$ & $-29.3(-31.1,-27.4)^{* *}$ & $50.1(20.7)$ & $32.0(21.2)$ & $-22.4(-24.9,-19.9)^{* *}$ & $<0.001$ & $<0.001$ \\
\hline Snoring while sleep (0-100) & $32.5(29.0)$ & $23.9(26.0)$ & $-7.1(-9.0,-5.2)^{* *}$ & $30.2(30.3)$ & $24.7(26.9)$ & $-4.8(-7.4,-2.2)^{* *}$ & 0.175 & 0.142 \\
\hline $\begin{array}{l}\text { Awakening short of breath } \\
(0-100)\end{array}$ & $39.9(22.8)$ & $17.7(19.6)$ & $-20.3(-22.2,-18.4)^{* *}$ & $30.6(23.9)$ & $20.9(22.9)$ & $-13.2(-15.7,-10.6)^{* *}$ & $<0.001$ & $<0.001$ \\
\hline Optimal sleep (0-1) & $0.19(0.39)$ & $0.65(0.48)$ & $0.46(0.41,0.51)^{* *}$ & $0.26(0.44)$ & $0.61(0.49)$ & $0.41(0.34,0.48)^{* *}$ & $<0.050$ & 0.268 \\
\hline Sleep quality (0-100) & $28.3(19.7)$ & $61.3(24.0)$ & $31.1(28.7,33.5)^{* *}$ & $35.7(22.6)$ & $55.1(26.1)$ & $22.8(19.5,26.1)^{* *}$ & $<0.001$ & $<0.001$ \\
\hline Daytime sleepiness (0-100) & $38.6(18.6)$ & $21.6(15.9)$ & $-16.0(-17.6,-14.4)^{* *}$ & $33.7(19.3)$ & $23.6(18.5)$ & $-12.5(-14.6,-10.3)^{* *}$ & $<0.001$ & 0.006 \\
\hline Sleep hours per night (0-24) & $5.53(1.25)$ & $6.91(1.05)$ & $1.27(1.16,1.38)^{* *}$ & $5.95(1.53)$ & $6.91(1.13)$ & $1.14(0.98,1.31)^{* *}$ & $<0.001$ & 0.170 \\
\hline $\begin{array}{l}\text { General index sleep problems } \\
(0-100)\end{array}$ & $55.0(15.0)$ & $27.0(16.0)$ & $-26.4(-28.1,-24.7)^{* *}$ & $47.5(16.8)$ & $31.2(19.1)$ & $-19.6(-22.0,-17.3)^{* *}$ & $<0.001$ & $<0.001$ \\
\hline \multicolumn{9}{|l|}{ EQ-5D questionnaire ${ }^{\mathrm{b}}$} \\
\hline \multirow[t]{2}{*}{ Mobility } & $1.40(0.50)$ & $1.22(0.44)$ & $-0.17(-0.21,-0.13)^{*}$ & $1.29(0.50)$ & $1.17(0.39)$ & $-0.17(-0.22,-0.12)^{*}$ & 0.002 & 0.922 \\
\hline & {$[39.4]$} & {$[21.4]$} & & {$[27.1]$} & {$[17.0]$} & & $<0.001$ & 0.375 \\
\hline \multirow[t]{2}{*}{ Personal care } & $1.26(0.45)$ & $1.09(0.29)$ & $-0.15(-0.18,-0.12)^{*}$ & $1.19(0.42)$ & $1.12(0.36)$ & $-0.12(-0.16,-0.08)^{*}$ & 0.027 & 0.174 \\
\hline & {$[26.0]$} & {$[8.7]$} & & {$[18.4]$} & {$[10.3]$} & & 0.032 & 0.196 \\
\hline \multirow[t]{2}{*}{ Activities of daily living } & $2.14(0.50)$ & $1.56(0.54)$ & $-0.52(-0.58,-0.47)^{* *}$ & $1.88(0.49)$ & $1.59(0.58)$ & $-0.43(-0.51,-0.36)^{* *}$ & $<0.001$ & 0.047 \\
\hline & [93.3] & {$[53.3]$} & & {$[81.5]$} & {$[54.7]$} & & $<0.001$ & 0.221 \\
\hline \multirow[t]{2}{*}{ Pain/discomfort } & $2.11(0.63)$ & $1.57(0.60)$ & $-0.51(-0.56,-0.45)^{* *}$ & $1.90(0.63)$ & $1.58(0.62)$ & $-0.42(-0.49,-0.35)^{* *}$ & $<0.001$ & 0.043 \\
\hline & {$[85.4]$} & {$[51.5]$} & & [74.6] & {$[50.8]$} & & $<0.001$ & 0.789 \\
\hline \multirow[t]{2}{*}{ Anxiety/depression } & $2.52(0.52)$ & $1.54(0.58)$ & $-0.94(-1.00,-0.64)^{* *}$ & $2.37(0.57)$ & $1.75(0.61)$ & $-0.72(-0.80,-0.64)^{* *}$ & 0.001 & $<0.001$ \\
\hline & {$[98.8]$} & {$[50.0]$} & & {$[95.8]$} & {$[65.7]$} & & 0.002 & $<0.001$ \\
\hline $\begin{array}{l}\text { Self-valuation quality of life } \\
\text { (EQ-VAS; 0-100) }\end{array}$ & $42.7(15.5)$ & $70.0(17.3)$ & $26.4(24.7,28.1)^{* *}$ & $48.9(15.7)$ & $65.5(18.3)$ & $19.4(17.1,21.6)^{* *}$ & $<0.001$ & $<0.001$ \\
\hline Health status (utility index; 0-1) & $0.40(0.30)$ & $0.78(0.25)$ & $0.36(0.34,0.38)^{* *}$ & $0.53(0.31)$ & $0.75(0.28)$ & $0.29(0.26,0.33)^{* *}$ & $<0.001$ & $<0.001$ \\
\hline
\end{tabular}

Values are expressed as mean (SD, standard deviation) or $95 \%$ confidence intervals (CI) unless otherwise stated.

${ }^{a}$ Comparison of the change from baseline between the two groups adjusted by baseline values and sex and age.

${ }^{b}$ The percentage of patients who still have problems (sum of categories 2 and 3 ) for each EQ-5D component are expressed within brackets.

$p<0.05,{ }^{*} p<0.01,{ }^{* *} p<0.001$ vs. baseline values.

of the total cost representing $58 \%$ of the total cost in the pregabalin group and 54\% in the UC group.

Health-care costs were significantly reduced in both cohorts yielding to similar 6-month costs; $€ 1,565 \pm 1,555$ pregabalin and $€ 1,406 \pm 1,611 \mathrm{UC}(p=0.777)$. At the 6month visit, medical visits were still responsible for most of the costs, but in a lower total percentage, representing now only the $30 \%$ and $41 \%$ of the total cost in the pregabalin and UC groups, respectively. The previously described reduction in the number of medical visits in both groups resulted in a significant reduction in the costs associated to this health resource $(€ 736$ in the pregabalin group and $€ 356$ in the UC group, $p<0.001$ in both groups), although mean adjusted pharmacologic cost increased significantly in both groups of patients: $€ 280$ in the pregabalin group and $€ 38$ in the UC group $(p<0.001)$.
The overall significant increase in the drug costs in the pregabalin group of patients was compensated by significant reductions in the number of visits and almost significant in the number of hospitalizations in this group of patients. Overall, these cost components resulted in a similarly meaningful reduction of total health-care costs both in adjunctive therapy with pregabalin and UC groups: -478 vs. $-446(p=0.777)$.

\section{Discussion}

In this paper, we report the significant improvement in symptoms observed in GAD patients with partial response to previous SSRI treatment when changed to adjunctive therapy with pregabalin in comparison with usual care. Under current European guidelines, it is recommended that pregabalin, SSRIs, and SNRIs are used as primary therapeutic options for the treatment of 


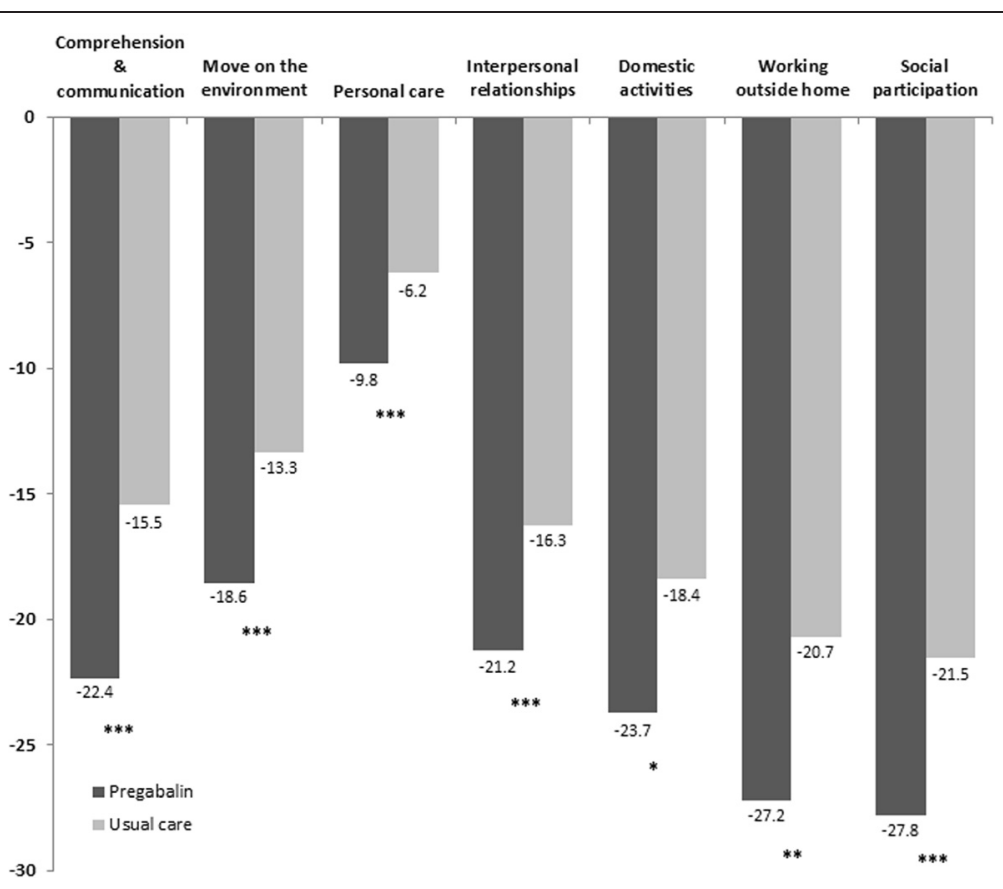

Figure 3 Adjusted mean reduction in patient disability reported WHO-DAS II components after 6 months of study in pregabalin and usual care groups. ${ }^{*} p<0.05 ;{ }^{*} p<0.01 ;{ }^{* *} p<0.001$ between groups adjusted by age, sex, and baseline values.

Table 3 Main pharmacological treatments during the study with pregabalin or usual care therapy

\begin{tabular}{|c|c|c|c|c|c|c|c|}
\hline \multirow{3}{*}{$\begin{array}{l}\text { Pharmacological } \\
\text { treatment }\end{array}$} & \multicolumn{3}{|c|}{ Pregabalin } & \multicolumn{3}{|l|}{ Usual care } & \multirow{3}{*}{$\begin{array}{l}p \text { (percentage } \\
\text { between } \\
\text { groups) }\end{array}$} \\
\hline & \multicolumn{3}{|l|}{$N=486$} & \multicolumn{3}{|l|}{$N=239$} & \\
\hline & $n(\%)$ & $\begin{array}{l}\text { Dose } \\
\text { mg/day (SD) }\end{array}$ & $\begin{array}{l}\text { Duration } \\
\text { months (SD) }\end{array}$ & $n(\%)$ & $\begin{array}{l}\text { Dose } \\
\text { mg/day (SD) }\end{array}$ & $\begin{array}{l}\text { Duration } \\
\text { months (SD) }\end{array}$ & \\
\hline Benzodiazepines & $326(67.1)$ & & & $162(67.8)$ & & & 0.867 \\
\hline Alprazolam & $136(28.0)$ & $2.0(1.3)$ & $4.7(2.0)$ & $50(20.9)$ & $2.0(1.4)$ & $4.7(1.9)$ & \\
\hline Diazepam & $58(11.9)$ & $10.2(7.1)$ & $4.4(2.0)$ & $26(10.9)$ & $12.9(6.8)$ & $5.1(1.7)$ & \\
\hline Lorazepam & $93(19.1)$ & $2.6(1.6)$ & $4.7(1.9)$ & $52(21.8)$ & $1.2(1.3)$ & $4.8(1.8)$ & \\
\hline Clorazepate dipotassium & $39(8.0)$ & $23.3(19.7)$ & $4.4(2.0)$ & $26(10.9)$ & $15.9(9.5)$ & $4.9(1.5)$ & \\
\hline Bromazepam & $21(4.3)$ & $5.7(6.4)$ & $4.8(1.9)$ & $10(4.2)$ & $4.7(2.1)$ & $4.9(1.5)$ & \\
\hline Clonazepam & $16(3.3)$ & $9.0(19.2)$ & $4.1(2.4)$ & $11(4.6)$ & $12.6(13.0)$ & $4.5(2.3)$ & \\
\hline Others & 75 (15.4) & - & - & $50(20.9)$ & - & - & \\
\hline SSRI & $331(68.1)$ & & & $162(67.8)$ & & & 0.933 \\
\hline Paroxetine & $121(24.9)$ & $24.5(8.9)$ & $5.0(1.9)$ & $62(25.9)$ & $25.6(10.8)$ & $5.3(1.6)$ & \\
\hline Escitalopram oxalate & $97(20.0)$ & $18.2(6.4)$ & $5.2(1.5)$ & $44(18.4)$ & $14.9(4.7)$ & $4.5(1.8)$ & \\
\hline Mirtazapine & $63(13.0)$ & $26.1(9.0)$ & $4.8(1.8)$ & $29(12.1)$ & $26.0(6.3)$ & $5.0(1.3)$ & \\
\hline Sertraline & $42(8.6)$ & $111.9(53.8)$ & $5.6(1.2)$ & $20(8.4)$ & $103.9(58.4)$ & $5.5(1.3)$ & \\
\hline Citalopram & $29(6.0)$ & $24.4(9.2)$ & $4.8(1.6)$ & $15(6.3)$ & $23.9(8.4)$ & $5.3(1.1)$ & \\
\hline Fluoxetine & $17(3.5)$ & $27.7(12.0)$ & $4.5(2.3)$ & $18(7.5)$ & $27.0(9.2)$ & $4.7(2.2)$ & \\
\hline Others & $12(2.5)$ & - & - & $13(5.5)$ & - & - & \\
\hline SNRI & $106(21.8)$ & & & $39(16.3)$ & & & 0.093 \\
\hline Venlafaxine hydrochloride & $61(12.6)$ & $155.4(53.9)$ & $5.3(1.4)$ & $26(10.9)$ & $148.0(47.3)$ & $5.6(1.0)$ & \\
\hline Duloxetine & $48(9.9)$ & 68.7 (26.9) & $5.5(1.7)$ & $13(5.4)$ & $61.8(21.0)$ & $4.9(1.6)$ & \\
\hline
\end{tabular}


Table 4 Health resources utilization and direct costs $(€)$ by treatment group

\begin{tabular}{|c|c|c|c|c|c|c|c|c|}
\hline & \multicolumn{3}{|l|}{ Pregabalin } & \multicolumn{3}{|l|}{ Usual care } & \multicolumn{2}{|c|}{$\begin{array}{l}p \text { between } \\
\text { groups }\end{array}$} \\
\hline & \multicolumn{3}{|l|}{$N=486$} & \multicolumn{3}{|l|}{$N=239$} & \multirow[b]{2}{*}{ Baseline } & \multirow[b]{2}{*}{ Final $^{\mathrm{a}}$} \\
\hline & Baseline & Final & Change $(95 \% \mathrm{Cl})$ & Baseline & Final & Change $(95 \% \mathrm{Cl})$ & & \\
\hline \multicolumn{9}{|l|}{ Resources } \\
\hline \multicolumn{9}{|l|}{ Non-pharmacological treatment ${ }^{b}$} \\
\hline Psychosocial therapy & $0.1(0.4)$ & $0.1(0.7)$ & $0.07(0.01,0.13)^{*}$ & $0.1(0.6)$ & $0.1(0.5)$ & $0.01(-0.06,0.09)$ & 0.122 & 0.226 \\
\hline Cognitive-behavioral therapies & $0.4(1.0)$ & $0.3(1.0)$ & $0.14(0.00,0.30)$ & $0.3(1.0)$ & $0.5(2.0)$ & $0.20(0.00,0.41)$ & 0.441 & 0.642 \\
\hline Supportive groups & $0.1(0.4)$ & $0.2(0.9)$ & $0.10(0.01,0.18)^{*}$ & $0.1(0.7)$ & $0.2(0.9)$ & $0.06(-0.06,0,18)$ & 0.476 & 0.588 \\
\hline Relaxation & $0.5(1.3)$ & $0.6(1.7)$ & $0.09(-0.06,0.25)$ & $0.5(1.5)$ & $0.7(2.3)$ & $0.13(-0.09,0.34)$ & 0.639 & 0.792 \\
\hline \multicolumn{9}{|l|}{ Number of medical visits ${ }^{c}$} \\
\hline Primary care & $11.9(13.9)$ & $2.4(3.7)$ & $-9.0(-9.5,-8.5)^{* * *}$ & $10.4(12.8)$ & $2.7(5.5)$ & $-8.3(-9.0,-7.6)^{* * *}$ & 0.015 & 0.100 \\
\hline Emergency department & $4.6(8.0)$ & $0.7(7.6)$ & $-3.9(-4.6,-3.2)^{* * *}$ & $3.7(6.9)$ & $0.3(0.8)$ & $-3.8(-4.8,-2.7)^{* * *}$ & 0.078 & 0.833 \\
\hline Psychologist & $3.0(7.2)$ & $1.8(4.6)$ & $-1.0(-1.5,-0.5)^{* * *}$ & $2.8(9.4)$ & $2.4(5.2)$ & $-0.9(-1.6,-0.2)^{* *}$ & 0.048 & 0.860 \\
\hline Psychiatrist & $5.9(6.4)$ & $3.9(3.7)$ & $-1.3(-1.7,-1.0)^{* * *}$ & $3.9(5.1)$ & $3.0(2.7)$ & $-1.9(-2.4,-1.3)^{* * *}$ & $<0.001$ & 0.113 \\
\hline Total medical visits & $25.4(22.0)$ & $8.7(11.2)$ & $-15.2(-16.3,-14.0)^{* * *}$ & $20.7(20.1)$ & $7.6(8.4)$ & $-14.9(-16.6,-13.2)^{* * *}$ & 0.001 & 0.754 \\
\hline Number of hospitalizations & $0.2(0.9)$ & $0.0(0.1)$ & $-0.10(-0.11,-0.09)^{* * *}$ & $0.1(0.6)$ & $0.0(0.1)$ & $-0.09(-0.10,-0.08)^{* * *}$ & 0.134 & 0.501 \\
\hline \multicolumn{9}{|l|}{ Costs (euros year 2009): } \\
\hline Total costs & $2,177(1,839)$ & $1,565(1,555)$ & $-478(-619,-338)^{* * *}$ & $1,724(2,059)$ & $1,406(1,611)$ & $-446(-635,-257)^{* * *}$ & $<0.001$ & 0.777 \\
\hline Drugs & $254(304)$ & $534(281)$ & $296(272,319)^{* * *}$ & $204(256)$ & $241(228)$ & $27(-5,58)$ & 0.001 & $<0.001$ \\
\hline Non-pharmacological treatment & $202(359)$ & $274(577)$ & $86(23,148)^{* *}$ & $191(379)$ & $282(748)$ & $88(3,172)^{*}$ & 0.115 & 0.969 \\
\hline Medical visits & $1,210(1,253)$ & $474(1,002)$ & $-660(-753,-567)^{* * *}$ & $936(1,136)$ & $580(893)$ & $-471(-596,-345)^{* * *}$ & $<0.001$ & 0.013 \\
\hline Hospitalization & $40(254)$ & $2(21)$ & $-28(-35,-21)^{* * *}$ & $14(149)$ & 9 (109) & $-17(-26,-8)^{* *}$ & 0.134 & 0.056 \\
\hline
\end{tabular}

Values are expressed as mean (SD, standard deviation) or $95 \%$ confidence intervals (Cl) unless otherwise stated.

${ }^{a}$ Comparison of the change from baseline between the two groups adjusted by baseline values and sex and age.

${ }^{b}$ Number of sessions per month.

In a 6-month period.

${ }^{*} p<0.05,{ }^{* *} p<0.01,{ }^{* * *} p<0.001$ vs. baseline values.

GAD. In addition, it is also recommended by the Spanish Ministry of Health [17] that benzodiazepines can be used for 2-4 weeks to prevent adverse events in the medium to long term as well as afflictive withdrawal symptomatology [12-15]. Under this premise, the understanding of current therapeutic options and their optimal utilization, under an efficient cost-consequence perspective, highlights the need for an appropriate estimation of economic consequences in the management of GAD patients. This estimation must take into account overall health-care resource and drug acquisition costs, especially when partial and/or partial responses to initial therapeutic options have been demonstrated [6].

To the best of our knowledge, our study presents the first analysis of clinical and economic consequences in GAD patients with partial response to previous treatment with SSRIs in routine medical care conditions. Our data adds support to previous results on the overall economic evaluation of different therapeutic options for the treatment of GAD patients in Spain [42,43]. Furthermore, this study evaluates the effective use of pregabalin as a treatment alternative when refractory outcomes have been demonstrated, i.e., with benzodiazepines [44].
In our study, as a result of the primary endpoint, we have observed that the addition of pregabalin to usual care leads to a significant reduction in anxiety and depression symptoms in comparison to usual care $(p<$ 0.0001 , in both cases). These reductions have been shown to be above or equal to the $50 \%$ of the baseline values in case of pregabalin. Significantly, it has to be stated that no differences in the anxiety and depression reductions were detected between sexes, thus dismissing a differential trend in terms of this fact. These trends are in accordance with a meta-analysis of eight published trials that evaluates the efficacy and tolerability of pregabalin in the treatment of GAD [45]. In this study, pregabalin treatment resulted in an overall lowered Hamilton Anxiety Rating Scale for anxiety scores within 1 week. The observed decrease in anxiety scores of our patients also confirms the recent findings by Rickels et al. that reported a significantly greater benefit for adjunctive pregabalin compared with placebo [46]. This randomized clinical trial of GAD patients with partial response to SSRI or SNRI described a mean reduction in HAM-A total score $(-7.6$ for pregabalin vs. -6.4 for placebo $(p<$ $0.05)$ ) during the 8 weeks of combination treatment [46]. 
When considering the depression symptoms in our study, the confirmed reductions sustain the initial observations of a pooled post hoc analysis that reflected pregabalin reduced associated symptoms of depression despite its dose [47]. In agreement with our results, a recent open-label trial [48] found statistically significant reductions $(p<0.001)$ in depression in older patients with GAD and comorbid depression from the fourth week of treatment. Global trends in amelioration of anxiety and depression outcomes were confirmed in our study by the significant change in the patient-reported Clinical Global Impression scale that benefits adjuvant pregabalin in GAD patients with partial response to SSRI with respect to UC $(p<0.005)$.

Comparable trends were detected for pregabalin adjuvant therapy in patient-reported outcomes concerning disability state and sleep quality. Significant differences in the reduction of all disability WHO-DAS II domains were probed at the end of the study and were found to favor pregabalin therapy, both in employed and unemployed patients $(p<0.0001$, in both cases). Improvements were substantial in cases of pregabalin treatment, with sleep amelioration and reduction in disability being of a magnitude above or equal to the $50 \%$ of the baseline values. The beneficial effects of pregabalin in disability status have been previously demonstrated in neuropathic pain patients evaluated through Sheehan Disability Inventory scale [49]. Besides, all items considered in the MOS-sleep scale, including average number of hours slept per day, presented significant higher improvements after the 6 months of the study for patients receiving pregabalin in comparison to UC. However, the only exceptions of this were the snoring score and the optimal sleep score that were lower with pregabalin but not significant. As suggested by a previous pooled analysis of seven studies of pregabalin therapy in GAD patients, $53 \%$ of the effect of pregabalin on sleep disturbance was due to a direct drug effect and $47 \%$ was due to an indirect effect mediated through prior reduction in anxiety symptom severity [50].

Improvement in sleep outcomes and reduction of daytime sleepiness in GAD patients treated with pregabalin has been also demonstrated in correlation with improvement in quality of life, according to subjective global measures, as well as with amelioration in functional impairment [50]. In our study sample of GAD patients with partial response to SSRI, pregabalin adjuvant therapy was associated with a differential 36\% improved benefits in self-valuation quality of life (EQ-5D visual analogue scale) and social tariff derived from such instrument in comparison with usual care. Furthermore, improvements in sleep and disability, together with major reductions in GAD symptoms, were translated to better outcomes in some dimensions of quality of life as described by the patient. Thus, in addition to anxiety/depression, patients receiving pregabalin also showed better activities of daily living and pain/discomfort in the EQ-5D questionnaire than subjects receiving usual care. These findings are coherent with previous publication [51].

Concerning therapeutic aspects and despite the fact that all the included patients in our analysis were partial responders for SSRIs, it should be noted than more than $2 / 3$ of the sample were still taking benzodiazepines during the last 6 months. This observation occurred independently of the pregabalin adjuvant incorporation, or lack thereof, in the usual care group. On the other hand, adding pregabalin was correlated with a near $1 / 3$ of reduction in benzodiazepines and SSRIs prescribed by physicians. It is important to consider that under this observation, psychiatrists could fulfil the recommended therapeutic goals related with benzodiazepines early by initiating GAD therapy with pregabalin in SSRI partial responders [52].

When comparing the results of our study with a previous Spanish economic evaluation of the annual cost of GAD, confirmed by DSM-IV criteria, the mean cost of GAD in primary care was estimated at $€ 686$, from which drug costs represented 59\% of the total costs [53]. This economic cost is higher than our present estimate where the pharmaceutical cost represents $34 \%$ of the overall costs in the pregabalin group and $17 \%$ in the usual care group. Overall, in our GAD patients sample with partial response to previous SSRIs, the mean total costs over 1 year decreased by $€ 478$ in the pregabalin group and by $€ 446$ in the usual care group. Although the pharmacological treatment costs per year were higher after pregabalin adjuvant incorporation ( $€ 534$ vs. $€ 241$ in UC, $p<$ $0.001)$, significant lower costs were detected for medical visits (€474 vs. $€ 580$ in $\mathrm{UC}, p=0.013)$ and an almost significant lower cost associated with hospitalizations ( $€ 2$ vs. $€ 9$ in UC, $p=0.056$ ). This framework results in a similar global health-care resource costs in both groups: $€ 1,565$ in the pregabalin group and $€ 1,406$ in the UC group $(p=0.777)$.

Main weaknesses of our work should also be noted. First, although some studies show that indirect costs resulting from associated loss of productivity double the usual direct costs of GAD [54,55], our study did not consider the indirect expenses associated with neither treatment nor the out-of-pocket costs as it was performed directly from the perspective of the National Health System. Second, the non-interventional design of the original data, the ADAN study, does not allow for any inference of direct causality between GAD treatment options and full costs of the associated patient's management. While this may be venturesome in terms of conclusive interpretations, our approach, based on realworld data, allows for health decision makers from the 
National Healthcare System to outline some interesting conclusions regarding the estimation of health-care resource utilization and its associated direct costs. Finally, the total study sample size included could be considered relevant in comparison with previous comparative cost analysis performed within the group of Spanish patients with refractory GAD $[10,56]$.

\section{Conclusions}

To conclude, our results suggested that initiating therapy with pregabalin in SSRI partial responders benefits anxiety and depression outcomes and does not imply significant increased direct costs when compared with the usual care treatment in a routine clinical practice basis. Moreover, the use of adjuvant pregabalin is associated with a lower use both of benzodiazepines and SSRIs as concomitant anxiolytic drugs. In spite of the limitations considered, the study showed that the significant higher pharmacological acquisition costs of pregabalin was compensated by improved clinical outcomes and higher reductions in non-pharmacological costs. Such a reduction in costs, such as medical visits and hospitalizations, could infer improved reductions in the overall management of GAD patients with partial response to SSRI from the National Health System perspective.

\begin{abstract}
Abbreviations
ADAN: Amplification of definition of anxiety; AT: Adjunctive therapy; APA: American Psychiatric Association; CGI-I: Clinical Global ImpressionImprovement; DSM-IV: Diagnostic and Statistical Manual of Mental Disorders version IV; EQ-5D: Euro Qol-5D; GAD: Generalized anxiety disorder; HAM-A: Hamilton Anxiety Rating Scale; MADRS: Montgomery Asberg Depression Rating Scale; MOS-sleep: Medical Outcome Study Sleep; PR: Partial response; QALY: Quality-adjusted life-year; SD: Standard deviation; SSRI: Selective serotonin reuptake inhibitor; SNRI: Selective serotonin-norepinephrine reuptake inhibitor; UC: Usual care; WHO-DAS II: World Health Organization Disability Assessment Schedule II; $\mathrm{Cl}$ : Confidence intervals.
\end{abstract}

\section{Competing interests}

The present work was carried out using the existing database of an observational, non-interventional study sponsored by Pfizer, S.L.U. Statistical analysis was carried out by Marta Figueras at TFS Develop and was funded by Pfizer Inc. Medical writing support was provided by Emili González-Pérez at TFS Develop and was funded by Pfizer Inc.

Dr. $J L$ Carrasco is member of national and international advisory boards at Janssen-Cilag, Pfizer, Lilly, Servier, Astra-Zeneca, Lundbeck and Abbot. Dr. E Álvarez has received consulting and educational honoraria from several pharmaceutical companies including Eli Lilly, Sanofi-Aventis, Lundbeck, Pfizer: and he has participated as main local investigator in clinical trials from Eli Lilly, Bristol-Myers and Sanofi-Aventis, and also as national coordinator of clinical trials from Servier and Lundbeck. Dr JM. Olivares is member of regional, national, and international advisory boards for Janssen-Cilag, Lilly, Astra-Zeneca and Bristol-Myers, and he has been involved in designing and/ or participating in clinical trials for Janssen-Cilag, Lilly, Astra-Zeneca, Pfizer, Lundbeck, Glaxo, and Bristol-Myers, and he has received educational grants for research, honoraria, and travel support for activities as a consultant/ advisor and lecturer/faculty member for Janssen-Cilag, Lilly, Astra-Zeneca, Pfizer, Lundbeck, Glaxo, Novartis and Bristol-Myers. Vanessa López-Gómez and Javier Rejas are employed in Pfizer, S.L.U. All other authors declare that they have no conflicts of interests as a consequence of this paper.

\section{Authors' contributions}

The authors of this manuscript state that all of the authors have seen and approved the submitted version of the manuscript and all authors have contributed substantially in the manuscript preparation, interpretation of results, or study design.

\section{Author details}

${ }^{1}$ Department of Psychiatry, Hospital de la Santa Creu i San Pau, Universitat Autónoma de Barcelona, CiberSam, Carrer Sant Quintí, 89, 08026 Barcelona, Spain. ${ }^{2}$ Department of Psychiatry, Hospital Meixoeiro, Complejo Hospitalario Universitario, Vigo, Spain. ${ }^{3}$ Department of Psychiatry, Hospital Clínico San Carlos, Universidad Complutense de Madrid, CiberSam, Madrid, Spain. ${ }^{4}$ Medical Department, Pfizer, S.L.U., Alcobendas, MD, Spain. ${ }^{5}$ Health Economics and Outcomes Research Department, Pfizer, S.L.U., Alcobendas, $\mathrm{MD}$, Spain.

Received: 19 November 2013 Accepted: 17 December 2014 Published online: 22 January 2015

\section{References}

1. Alonso J, Angermeyer MC, Bernert S, Bruffaerts R, Brugha TS, Bryson $\mathrm{H}$, et al. Prevalence of mental disorders in Europe: results from the European Study of the Epidemiology of Mental Disorders (ESEMeD) project. Acta Psychiatr Scand Suppl. 2004;420:21-7.

2. Ansseau M, Fischler B, Dierick M, Mignon A, Leyman S. Prevalence and impact of generalized anxiety disorder and major depression in primary care in Belgium and Luxemburg: the GADIS study. Eur Psychiatry. 2005:20:229-35.

3. Wetherell JL, Birchler GD, Ramsdell J, Unutzer J. Screening for generalized anxiety disorder in geriatric primary care patients. Int J Geriatr Psychiatry. 2007:22:115-23.

4. Kessler RC, Berglund P, Demler O, Jin R, Merikangas KR, Walters EE. Lifetime prevalence and age-of-onset distributions of DSM-IV disorders in the National Comorbidity Survey Replication. Arch Gen Psychiatry. 2005:62:593-602.

5. Haro JM, Palacin C, Vilagut G, Martinez M, Bernal M, Luque I, et al. Prevalence of mental disorders and associated factors: results from the ESEMeD-Spain study. Med Clin (Barc). 2006;126:445-51.

6. Konnopka A, Leichsenring F, Leibing E, Konig HH. Cost-of-illness studies and cost-effectiveness analyses in anxiety disorders: a systematic review. J Affect Disord. 2009;114:14-31.

7. Hoffman DL, Dukes EM, Wittchen HU. Human and economic burden of generalized anxiety disorder. Depress Anxiety. 2008;25:72-90.

8. Kertz SJ, Woodruff-Borden J. Human and economic burden of GAD, subthreshold GAD, and worry in a primary care sample. J Clin Psychol Med Settings. 2011;18:281-90.

9. Sicras-Mainar A, Blanca-Tamayo M, Navarro-Artieda R, Rejas-Gutierrez J. Use of resources and costs profile in patients with fibromyalgia or generalized anxiety disorder in primary care settings. Aten Primaria. 2009;41:77-84.

10. Rovira J, Albarracin G, Salvador L, Rejas J, Sanchez-lriso E, Cabases JM. The cost of generalized anxiety disorder in primary care settings: results of the ANCORA study. Community Ment Health J. 2012;48:372-83.

11. Gould RA, Otto MW, Pollack MH, Yap L. Cognitive behavioral and pharmacological treatment of generalized anxiety disorder: a preliminary meta-analysis. Behav Ther. 1997;28:285-305.

12. Martin $J \mathrm{~L}$, Sainz-Pardo M, Furukawa TA, Martin-Sanchez E, Seoane T, Galan C. Benzodiazepines in generalized anxiety disorder: heterogeneity of outcomes based on a systematic review and meta-analysis of clinical trials. J Psychopharmacol. 2007;21:774-82.

13. Rickels K, Schweizer E, Csanalosi I, Case WG, Chung H. Long-term treatment of anxiety and risk of withdrawal. Prospective comparison of clorazepate and buspirone. Arch Gen Psychiatry. 1988;45:444-50.

14. Tyrer $P$, Owen R, Dawling $S$. Gradual withdrawal of diazepam after long-term therapy. Lancet. 1983;1:1402-6.

15. Baldwin DS, Polkinghorn C. Evidence-based pharmacotherapy of generalized anxiety disorder. Int J Neuropsychopharmacol. 2005;8:293-2.

16. Guideline Working Group for the Treatment of Patients with Anxiety Disorders in Primary Care, Health Technology Assessment Unit, Laín Entralgo Agency, Ministry of Health, Social Services and Equality (Spain). 2008: Clinical practice guideline for treatment of patients with anxiety 
disorders in primary care, [http://www.guiasalud.es/GPC/GPC_430_Ansiedad_ Lain_Entr_compl.pdf]

17. Vicens Caldentey C, Fiol Gelabert F, Gonzalez Garrido E, Martinez Ojeda E, Mateu Sabater C, Llobera CJ. Long-term effectiveness of an intervention to discontinue chronic benzodiazepine use. Actas Esp Psiquiatr. 2008;36:295-8.

18. Bandelow B, Zohar J, Hollander E, Kasper S, Moiler HJ, Wfsbp Task Force on Treatment Guidelines for Anxiety O-CaPSD. World federation of societies of biological psychiatry (WFSBPJ) guidelines for the pharmacological treatment of anxiety, obsessive-compulsive and posttraumatic stress disorders. World J Biol Psychiatr. 2002;3:171-99.

19. Keck Jr PE, Strawn JR, Mcelroy SL. Pharmacologic treatment considerations in co-occurring bipolar and anxiety disorders. J Clin Psychiatry. 2006;67 Suppl 1:8-15.

20. McElroy SL, Altshuler LL, Suppes T, Keck Jr PE, Frye MA, Denicoff KD, et al. Axis I psychiatric comorbidity and its relationship to historical illness variables in 288 patients with bipolar disorder. Am J Psychiatry. 2001;158:420-6.

21. Simon NM, Otto MW, Wisniewski SR, Fossey M, Sagduyu K, Frank E, et al. Anxiety disorder comorbidity in bipolar disorder patients: data from the first 500 participants in the Systematic Treatment Enhancement Program for Bipolar Disorder (STEP-BD). Am J Psychiatry. 2004;161:2222-9.

22. Schifano F. Misuse and abuse of pregabalin and gabapentin: cause for concern? CNS Drugs. 2014;28:491-6. doi:10.1007/s40263-014-0164-4.

23. Martinotti G, Lupi M, Sarchione F, Santacroce R, Salone A, De Berardis D, et al. The potential of pregabalin in neurology, psychiatry and addiction: a qualitative overview. Curr Pharm Des. 2013;19:6367-74.

24. Feltner DE, Crockatt JG, Dubovsky SJ, Cohn CK, Shrivastava RK, Targum SD, et al. A randomized, double-blind, placebo-controlled, fixed-dose, multicenter study of pregabalin in patients with generalized anxiety disorder. J Clin Psychopharmacol. 2003;23:240-49.

25. Rickels K, Pollack MH, Feltner DE, Lydiard RB, Zimbroff DL, Bielski RJ, et al. Pregabalin for treatment of generalized anxiety disorder: a 4-week, multicenter, double-blind, placebo-controlled trial of pregabalin and alprazolam. Arch Gen Psychiatry. 2005;62:1022-30.

26. Montgomery SA, Tobias K, Zornberg GL, Kasper S, Pande AC. Efficacy and safety of pregabalin in the treatment of generalized anxiety disorder: a 6-week, multicenter, randomized, double-blind, placebo-controlled comparison of pregabalin and venlafaxine. J Clin Psychiatry. 2006;67:771-82.

27. Pande AC, Crockatt JG, Feltner DE, Janney CA, Smith WT, Weisler R, et al. Pregabalin in generalized anxiety disorder: a placebo-controlled trial. Am J Psychiatry. 2003;160:533-40.

28. Simes RJ. Clinical trials and "real-world" medicine. Trial evidence best informs real-world medicine when it is relevant to the clinical problem. Med J Aust. 2002;177:410-11.

29. Alvarez E, Carrasco JL, Olivares JM, Lopez-Gomez V, Vilardaga I, Perez M. Broadening of generalized anxiety disorders definition does not affect the response to psychiatric care: findings from the observational ADAN study. Clin Pract Epidemiol Ment Health. 2012;8:158-68.

30. Hamilton M. The assessment of anxiety states by rating. $\mathrm{Br} J$ Med Psychol. 1959;32:50-5.

31. Lobo A, Chamorro L, Luque A, Dal-Re R, Badia X, Baró E, et al. Validation of the Spanish versions of the Montgomery-Asberg depression and Hamilton anxiety rating scales. Med Clin (Barc). 2002;118:493-9.

32. Guy W. Clinical Global Impressions. In: HEALTH, N I F M, editor. ECDEU Assessment Manual for Psycopharmacology Revised. Rockville, MD: U.S. Department of Health, Education, and Welfare; 1976.

33. Oblikue. Base de Datos de Costes Sanitarios eSALUD. Barcelona (Spain); 2012. [http://www.oblikue.com/bddcostes/]

34. INE 2012. Spanish Statistical Office. [http://www.ine.es/]

35. Busner J, Targum SD. The clinical global impressions scale: applying a research tool in clinical practice. Psychiatry (Edgmont). 2007;4:28-37.

36. Williams JB, Kobak KA. Development and reliability of a structured interview guide for the Montgomery Asberg Depression Rating Scale (SIGMA). Br J Psychiatry. 2008;192:52-8

37. Allen RP, Kosinski M, Hill-Zabala CE, Calloway MO. Psychometric evaluation and tests of validity of the medical outcomes study 12-item sleep scale (MOS sleep). Sleep Med. 2009;10:531-9.

38. Luciano JV, Ayuso-Mateos JL, Aguado J, Fernandez A, Serrano-Blanco A, Roca $\mathrm{M}$, et al. The 12-item world health organization disability assessment schedule II (WHO-DAS II): a nonparametric item response analysis. BMC Med Res Methodol. 2010;10:45.
39. Badia X, Roset M, Montserrat S, Herdman M, Segura A. The Spanish version of EuroQol: a description and its applications. European quality of life scale. Med Clin (Barc). 1999;112(1):79-85.

40. Lobo A, Chamorro L, Luque A, Dal-Ré R, Badía X, Baró E. Validación de las versiones en español de la Montgomery-Asberg Depression Scale y la Hamilton Anxiety Rating Scale para la evaluación de la depresión y de la ansiedad. Med Clin (Barc). 2002;118:493-9.

41. Thompson SG, Barber JA. How should cost data in pragmatic randomised trials be analysed? BMJ. 2000;320:1197-200.

42. Vera-Llonch M, Dukes E, Rejas J, Sofrygin O, Mychaskiw M, Oster G. Cost-effectiveness of pregabalin versus venlafaxine in the treatment of generalized anxiety disorder: findings from a Spanish perspective. Eur J Health Econ. 2010;11:35-44.

43. Consejo General de Colegios Farmacéuticos. Base de datos de medicamentos (2012). [http://www.portalfarma.com/Paginas/default.aspx]

44. De Salas-Cansado M, Alvarez E, Olivares JM, Carrasco JL, Ferro MB, Rejas J. Modelling the cost-effectiveness of pregabalin versus usual care in daily practice in the treatment of refractory generalised anxiety disorder in Spain. Soc Psychiatry Psychiatr Epidemiol. 2013;48:985-96.

45. Wensel TM, Powe KW, Cates ME. Pregabalin for the treatment of generalized anxiety disorder. Ann Pharmacother. 2012;46:424-29.

46. Rickels K, Shiovitz TM, Ramey TS, Weaver JJ, Knapp LE, Miceli JJ. Adjunctive therapy with pregabalin in generalized anxiety disorder patients with partial response to SSRI or SNRI treatment. Int Clin Psychopharmacol. 2012;27:142-50.

47. Stein DJ, Baldwin DS, Baldinetti F, Mandel F. Efficacy of pregabalin in depressive symptoms associated with generalized anxiety disorder: a pooled analysis of 6 studies. Eur Neuropsychopharmacol. 2008;18:422-30.

48. Karaiskos D, Pappa D, Tzavellas E, Siarkos K, Katirtzoglou E, Papadimitriou $\mathrm{GN}$, et al. Pregabalin augmentation of antidepressants in older patients with comorbid depression and generalized anxiety disorder-an open-label study. Int J Geriatr Psychiatry. 2013;28:100-5.

49. Navarro A, Saldana MT, Perez C, Torrades S, Rejas J. Patient-reported outcomes in subjects with neuropathic pain receiving pregabalin: evidence from medical practice in primary care settings. Pain Med. 2010;11:719-31.

50. Holsboer-Trachsler E, Prieto R. Effects of pregabalin on sleep in generalized anxiety disorder. Int J Neuropsychopharmacol. 2013;16:925-36.

51. De Salas-Cansado M, Olivares JM, Alvarez E, Carrasco JL, Barrueta A, Rejas J. Pregabalin versus SSRIs and SNRIs in benzodiazepine-refractory outpatients with generalized anxiety disorder: a post hoc cost-effectiveness analysis in usual medical practice in Spain. Clinicoecon Outcomes Res. 2012;4:157-68.

52. Ballenger JC, Davidson JR, Lecrubier Y, Nutt DJ, Borkovec TD, Rickels K, et al. Consensus statement on generalized anxiety disorder from the international consensus group on depression and anxiety. J Clin Psychiatry. 2001;62 Suppl 11:53-8.

53. Sicras-Mainar A, Blanca-Tamayo M, Navarro-Artieda R, Pizarro-Paixa I, Gomez-Lus CS. Influence of morbidity and the use of health resources in patients who require care for generalised anxiety disorder in the primary health care setting. Aten Primaria. 2008:40:603-10.

54. Iskedjian M, Walker JH, Bereza BG, Le Melledo JM, Einarson TR. Costeffectiveness of escitalopram for generalized anxiety disorder in Canada. Curr Med Res Opin. 2008;24:1539-48.

55. Olfson M, Gameroff MJ. Generalized anxiety disorder, somatic pain and health care costs. Gen Hosp Psychiatry. 2007;29:310-6.

56. Albarracin G, Rovira J, Carreras L, Rejas J. Economic and epidemiologic aspects of generalized anxiety disorder: a review of the literature. Actas Esp Psiquiatr. 2008;36:165-76. 